\title{
Desarrollando Fondos de Conocimiento: Prácticas \\ Educativas Socialmente Justas en el Contexto Español
}

\section{Developing Funds of Knowledge: Social Justice Educational \\ Practices in the Spanish Context}

\author{
David Subero * \\ Universitat de Girona, España
}

\begin{abstract}
DESCRIPTORES:
Fondos de conocimiento

Justicia social

Educación inclusiva

Relaciones familia-

escuela

Diversidad

\section{RESUMEN :}

Los fondos de conocimiento ( $\mathrm{FdC}$ ), desde su origen a principios de la década de los noventa, han tenido como objetivo el desarrollo de prácticas educativas escolares sensibles y contextualizadas a las experiencias familiares del alumnado culturalmente diversos. Desde un marco sociocultural, el enfoque se ha convertido en un referente en prácticas de justicia social educativa asumiendo una visión crítica y poniendo en el centro de su acción transformadora la lucha contra las desigualdades estructurales, las relaciones de poder y la perspectiva del déficit cultural en educación como discurso dominante. A su vez, propone un marco teórico-metodológico para recuperar la dignidad y la representatividad de las familias en las escuelas, a través de sus conocimientos, recursos y fortalezas. El procedimiento consiste en visitar a las familias por parte de los docentes y recoger los fondos de conocimiento a través de entrevistas y técnicas etnográficas, para luego desarrollar unidades didácticas en el contexto escolar. A continuación, se presentan tres experiencias educativas en el contexto español guiadas por el enfoque de los FdC, en dónde se destacan prácticas pedagógicas originales, redistributivas y representativas con el objetivo de establecer actuaciones educativas culturalmente congruentes y avanzar hacia la inclusión.
\end{abstract}

\section{KEYWORDS: \\ Funds of knowledge \\ Social justice \\ Inclusive education \\ Family school \\ partnership \\ Diversity}

\begin{abstract}
:
The Funds of Knowledge (FoK), since their origin in the early nineties, have aimed at developing sensitive and contextualized school educational practices to the family experiences of culturally diverse students. From a sociocultural approach, the program has become a benchmark in educational social justice practices, assuming a critical approach and placing structural inequalities, power relations and the perspective of cultural deficit in education at the center of its transformative action. In turn, it proposes a theoretical-methodological framework to recover the dignity and representativeness of families in schools, through their knowledge, resources and strengths. The procedure consists of visiting the families by the teachers and collecting the funds of knowledge through interviews and ethnographic techniques, to then develop didactic units in the school context. Next, three educational experiences in the Spanish context are presented, guided by the FoK approach, where original, redistributive and representative pedagogical practices are highlighted with the aim of improving educational processes of cultural congruence and the development of inclusive cultures.
\end{abstract}




\section{Introducción}

Hay una realidad cada vez más evidente en las aulas y es el aumento de la diversidad étnica y cultural del alumnado. Este hecho se manifiesta de tal modo que la escuela es un reflejo de lo que en la sociedad ocurre, y nos encontramos ante tendencias sociales y culturales caracterizadas por: 1) una nueva modernidad (Bauman, 2017; Pardo, 2016); 2) el avance de la globalización económica que se manifiesta en una homogenización del capital, pero que a su vez acentúa una diversidad cultural a la cual no se da respuesta (Darling-Hammond, 2010); 3) la expansión de la sociedad digital, que provoca la movilidad de las personas hacia lugares en donde puedan estar en contacto con redes de información (Castells, 1998), ya que hoy en día, no estar conectado significa estar excluido (Han, 2014); 4) el avance hacia el desarrollo casi exclusivo de competencias individuales y un estilo de vida basado en la competencia (Esteban-Guitart y Ratner, 2011) y 5) la continua vulneración de derechos que se siguen llevando a cabo hacia los más vulnerables (Nussbaum, 2015).

Es decir, esta es la cara menos agradable de la economía global del conocimiento que se caracteriza por continuar manteniendo el sistema social y la forma de vida del capitalismo del consumidor. Un capitalismo que se ha adaptado a las nuevas realidades y contextos, pero sigue manteniendo la producción, la distribución y el consumo de los bienes en manos de inversores de capital privado que se lucran en el libre mercado (Ratner, 2011). Esta realidad, en una economía global del conocimiento que selecciona a las personas como válidas o no válidas para su sistema de producción, provoca la creación de grandes bolsas de "precariado" (Standing, 2013) que no tienen lugar en el sistema y quedan excluidas de él. Esto acrecienta la sensación de incertidumbre y falta de control de las personas sobre su propia vida, la fragilidad de los trabajos que un día están y otros desaparecen, el aumento de desigualdades entre los ricos y pobres y la necesidad de las personas de dirigirse hacia dónde la seguridad y la estabilidad sean mayores en un mundo cambiante y líquido (Bauman, 2017).

Por la necesidad de las poblaciones de buscar nuevas oportunidades, en las últimas décadas, ha habido un aumento de los flujos migratorios mundiales (Sassen, 2010), en especial hacia lugares como Asia, Estados Unidos y Europa. Por lo que respecta al contexto español, ha habido un crecimiento progresivo de población inmigrante, también en sus aulas. La presencia de lenguas, identidades, religiones y tradiciones familiares heterogéneas ha tenido un impacto evidente en las escuelas, transformando sus poblaciones escolares. Sin ir más lejos, el porcentaje de estudiantes de origen extranjero ha crecido del $1.4 \%$ en el año 2000 al 8.8\% en el año 2019 (MECD, 2018).

En España, además del desafío de acoger al alumnado recién llegado en las escuelas, se ha ido añadiendo otro tipo de demandas caracterizadas por prácticas pedagógicas de calidad, así como la garantía del éxito educativo para todos. Esta última demanda se erige como un tema central de debate y un reto para los países más desarrollados (OECD, 2018). Sin ir más lejos, la misma UNESCO (2000) afirma que la participación activa, igualitaria y equitativa de todo el alumnado es fundamental para la construcción de una sociedad justa dentro de la sociedad del conocimiento actual.

Sin embargo, según los últimos informes PISA (OECD, 2019), existe una brecha que es consistente a lo largo de los últimos años, entre el rendimiento académico del alumnado infrarrepresentado ${ }^{1}$ y el alumnado de clase media estándar en el contexto educativo español. De hecho, según PISA (OECD, 2016), los estudiantes infrarrepresentados no solamente tienen un rendimiento escolar menor, sino que además tienen tendencia a dejar de ir a clase o llegar tarde, así como bajos niveles de compromiso y motivación en relación al aprendizaje escolar.

Como han resaltado algunos autores (Esteban-Guitart et al., 2019), en base a los datos ofrecidos por los informes PISA, ni las variables socioeconómicas ni la falta de familiaridad con la lengua vehicular en la escuela, justifican el bajo rendimiento y los hándicaps que se apuntan en el párrafo anterior. A su vez, rechazamos la tesis del déficit cultural (Valencia, 2010), muy extendidas en occidente, en dónde el alumno

1 Aquellos alumnos y alumnas que tienen una mayor dificultad en ser reconocidos en las practicas académicas por sus bajos ingresos, grupo étnico minoritario o bien de origen extranjero (Rios-Aguilar et al., 2011 p. 179). Por ende, en esta definición también está incluido el alumnado de origen inmigrante tanto de primera como de segunda generación. 
infrarrepresentado no aprende porque sus familias no tienen experiencias de aprendizaje lo suficientemente relevantes, lo que lleva a carencias en la socialización y en las prácticas académicas, que la escuela ha de compensar con más intensidad. En estos casos, no son raros los espacios escolares abiertos a las familias para decir cómo y de qué manera tienen que educar a sus hijos, dejando muchas veces de lado sus propias experiencias culturales y saberes familiares acumulados, para así educarles de forma "normalizada" o estándar.

Por su parte, autores como Belavi y Murillo (2020), plantean nuevas formas de reconsiderar la escuela a partir de cambios en: 1) la distribución de oportunidades y beneficios de la educación y 2) el reconocimiento de valores culturales y la diversidad social de nuestro alumnado y sus familias, que están fuertemente ligados con la justicia social educativa. Por justicia social educativa entendemos prácticas educativas diversas y equitativas que luchan contra las desigualdades, injusticias sociales y opresiones (Erchick y Tyson, 2013; Subero et al., 2015). En primer lugar, incidimos en el hecho que no todos los estudiantes parten de la misma línea de salida, y eso hace que las oportunidades y los recursos estén distribuidos de forma injusta y poco equitativa. De este modo, es necesario llevar a la práctica acciones educativas teniendo en especial consideración las necesidades del alumnado infrarrepresentado, con el objetivo que la educación de calidad sea algo de todos, y no de unos pocos. En segundo lugar, se considera que las escuelas, tradicionalmente, han mantenido un tipo de actividad estándar que va desde los discursos, las formas de relación, las pedagogías y el currículum prevalente aislando (o rechazando) de forma directa (o indirecta) todos aquellos discursos, formas de relación y prácticas culturales y educativas que no predominan en esta estructura, generando evidencias claras de injusticia social. En otras palabras, en las escuelas, la jerarquía de valores culturales dominantes no ha tenido en cuenta las identidades, perspectivas y estilos de vida de las diferentes diversidades. Por ello, se sugieren programas educativos que hagan visibles, respeten y operen con las culturas que los estudiantes infrarrepresentados traen consigo al sistema escolar (Gay, 2018).

A continuación se pueden resumir algunas características que tienen los programas educativos basados en la justicia social:

- Este tipo de programas suelen tener una implicación de concientización y empoderamiento en el alumnado y en las familias. Es decir, por una parte, desarrollan la capacidad de análisis crítico sobre su situación de opresión en los espacios educativos (Freire, 1998) y, por la otra, son capaces de cambiar su situación (intelectual, social, emocional y política) a partir del uso de sus referentes culturales en la enseñanza (Ladson-Billings, 1995).

- Cuando los programas consiguen enlazar la individualidad de los estudiantes con su identidad étnica y su socialización cultural (Gay, 2018), hay mejoras en el rendimiento y éxito académico del alumnado.

- En este tipo de programas los profesores juegan un papel fundamental en el proceso. Es decir, los docentes han de ser expertos y familiarizarse con la cultura de origen de su alumnado y familias, para de este modo poder ser referentes claros en las prácticas pedagógicas y combatir prejuicios y estereotipos (Hollie, 2018).

- Los programas van más allá de una propuesta académica al uso. Es decir, son programas que, de una forma holística, incorporan dimensiones tales como lo emocional y lo psicológico. Sólo de este modo es posible reconocer la singularidad cultural de los estudiantes y familias, así como conocer sus experiencias y conocimientos. Se entiende que implicando a las familias y al alumnado en el proceso, es posible proponer aprendizajes más relevantes y eficaces. Para ello, se modifican las relaciones tradicionales en dónde el docente es el transmisor de unos supuestos conocimientos establecidos y validados por la autoridad educativa, para hacer que las familias y los estudiantes "se conviertan en estudiosos de la diversidad cultural y generen su propio contenido en el currículum” (Gay, 2018, p. 144).

En coherencia con las nociones anteriormente mencionadas, se propone el enfoque de los fondos de conocimiento (Llopart y Esteban-Guitart, 2018; Moll, 2019) como una propuesta capaz de modificar las relaciones familia y escuela hacia mayores cotas de concienciación, poniendo en el centro del proyecto educativo los hogares del alumnado infrarrepresentados. Además, su despliegue en las escuelas tiene como finalidad recono- 
cer las prácticas culturales familiares como valiosas, generar cambios en el docente rompiendo con prejuicios y/o estereotipos y establecer espacios de confianza mutua implicando a las familias en el proceso de aprender.

\section{Los fondos de conocimiento}

Los fondos de conocimiento tienen su origen en la antropología y aparecen por primera vez en los trabajos realizados en la Universidad de Arizona (USA) por Vélez-Ibáñez y Greenberg (1992). Los autores pusieron el foco en las relaciones económicas que tenían los migrantes mexicanos que residían en Estados Unidos, y la forma en que estas relaciones económicas se desarrollaban. Las primeras conclusiones fueron que las relaciones de confianza mutua y los lazos sociales eran fundamentales para hacer frente a las incertidumbres que emergen en una situación de tránsito como el fenómeno migratorio. Además, estas relaciones de confianza mutua estaban constituidas por una serie de conocimientos y habilidades de gran importancia para las familias.

Los fondos de conocimiento, rápidamente tuvieron su relevancia en el campo de la educación de la mano del psicólogo educativo Luis Moll y colaboradores con la creación y desarrollo del "Proyecto Fondos de Conocimiento para la Enseñanza" (Gonzalez, Moll y Amanti, 2005). El enfoque aparece como parte de un conjunto de propuestas basadas en la justicia social educativa dirigidas a transformar el currículum y el aprendizaje de tal modo que fueran más equitativos y significativos para los estudiantes de comunidades históricamente marginalizadas e infrarrepresentadas; en este caso en familias mexicano-americanas de la ciudad de Tucson.

La aproximación de los fondos de conocimiento (Moll, 2014, 2019) ha sido una alternativa creativa y contextualizada que, lejos de entender a las familias del alumnado culturalmente diverso como carente de conocimientos y experiencias válidas para la escuela, afirman que "todas las familias cuentan con cuerpos de conocimiento y habilidades históricamente acumuladas y culturalmente desarrolladas esenciales para el buen funcionamiento y bienestar de los hogares o los miembros de la familia" (Moll, 2014 p. 25). Es decir, los fondos de conocimiento se basan en la premisa que todos los estudiantes y sus familias son valiosas y acumulan conocimientos, habilidades y recursos culturales. En este contexto, se tiene en cuenta las experiencias familiares y las prácticas culturales como repositorios de conocimiento.

Los tres objetivos del enfoque son los siguientes: 1) La mejora del rendimiento educativo del alumnado infrarrepresentado; 2) La mejora de las relaciones entre la familia y la escuela (a través de procesos de confianza mutua, en dónde el profesorado hace emerger los fondos de conocimiento de familias y alumnado); 3) La modificación de la práctica docente a través del diseño de nuevas unidades didácticas y cambios en el currículum a partir de los fondos de conocimiento identificados. De este modo, se pretende que la institución escolar esté conectada con aspectos importantes de la vida del alumnado como las relaciones familia-escuela, en especial con aquellas familias que cuentan con menor representatividad en las prácticas educativas escolares.

Cuadro 1

Fases de Programas basados en Fondos de Conocimiento

Fases Descripción

1. Explicación del Programa (Profesores e Investigadores)

Se introduce la teoría de fondos y se enseñan técnicas etnográficas (entrevista, recogida de datos a partir de la observación).

2. Visita a las Familias (Profesores)

3. Grupos de Estudio

(Profesores e Investigadores)

4. Unidades didácticas basadas en fondos de conocimiento (Profesores)
Se accede al domicilio, se observa el contexto y se realiza la entrevista.

Se comparten los fondos de conocimiento entre el grupo de profesores y se elaboran las secuencias didácticas.

Se llevan a cabo las actividades en el aula incorporando los nuevos fondos.

Nota. Elaboración propia a partir de Gonzalez, Moll y Amanti (2005). 
A grandes rasgos, los programas educativos basados en el enfoque de los fondos de conocimiento se desarrollan de la forma siguiente (Cuadro 1). En un principio, con el objetivo de mejorar los procesos de aprendizaje de los estudiantes infrarrepresentados, se sugiere, desde un marco sociocultural, una vía para reconfigurar la enseñanza y el currículum escolar basada en las prácticas culturales y las experiencias familiares. Se sugiere, en esta primera fase, introducir el marco teórico y metodológico de los fondos a los docentes participantes en el proyecto para familiarizarlos con las estrategias y formas de entender el enfoque. Seguidamente, los profesores visitan los hogares de los estudiantes, con el objetivo de documentar empíricamente los fondos de conocimientos particulares y así poderlos usar pedagógicamente en las aulas. Al mismo tiempo que se llevan a cabo estas visitas a las familias, emerge una relación de colaboración más estrecha basada en la confianza mutua. En estas visitas, se hace uso de una serie de técnicas etnográficas para recoger las informaciones a través de experiencias de vida y conocimientos acumulados. Los profesores preguntan sobre la historia familiar, sobre las historias laborales, las aficiones, actividades e intereses, prácticas de alfabetización, entre otras. El objetivo es hacer evidentes los fondos de conocimiento en el núcleo familiar. Los grupos de estudio pueden ser considerados como espacios mediadores donde tanto los docentes como los investigadores comparten significados y recursos para teorizar sobre los fondos familiares de los estudiantes, así como planificar un mejor acondicionamiento en el currículum escolar y en las prácticas educativas (Moll, 2014; Esteban-Guitart et al., 2018). Una vez identificados, los profesores incorporan estas habilidades y conocimientos en las actividades del aula, estableciendo de este modo continuidades entre los fondos de conocimientos acumulados por las familias, el currículum escolar y las prácticas de enseñanza en la escuela.

\section{Proyectos}

A continuación, voy a hacer una revisión de tres estudios llevados a cabo en el contexto español basados en fondos de conocimiento, en los cuales, aunque partiendo de grupos infrarrepresentados como foco, el contexto sociocultural y el desenlace de los procesos y prácticas educativas son a su vez genuinos y originales. De este modo, los investigadores reorientan el concepto de los fondos de conocimiento para abordar cuestiones relacionadas con grupos culturales específicos, tales como las familias de origen marroquí y las familias gitanas, así como el proyecto Girona, que engloba una intervención del alumnado de diversas procedencias abriendo el abanico de la intervención a la diversidad cultural y familiar. Los proyectos fueron seleccionados por su variedad en la tipología de participantes y por las diferentes formas de acercarse al enfoque de los fondos de conocimiento a partir de las dinámicas socioculturales que se encuentran en la familia y en vida de la comunidad. Es decir, por una parte, cada una de las tres experiencias tiene como foco alumnado de grupo minoritario diferente (familia marroquí en el primero, familia gambiana en el segundo y familia gitana en el tercero). Por la otra, los dos primeros ejemplos se llevan a cabo a través de la metodología de visitas a los hogares por parte de los docentes, mientras que la tercera experiencia incorpora un tercer actor que hace de mediador entre los docentes y las familias en el desarrollo del proyecto.

Antes de continuar, sin embargo, es necesario hacer un pequeño inciso sobre el desarrollo educativo del alumnado de familias inmigrantes y familias gitanas en el contexto español. Para ello, es necesario contextualizar el hecho de que la escolarización del alumnado inmigrante y/o de minorías étnicas (tales como la gitana), se caracteriza por una ausencia sostenida de éxito escolar en España (Lalueza y Crespo, 2009). Este hecho se sustenta en la perspectiva del déficit que entiende al alumnado responsable de su fracaso en la escuela. Por el contrario, desde un marco sociocultural y ecológico se entiende necesario involucrar activamente a la escuela en la responsabilidad del fracaso del alumnado, proponiendo a su vez opciones de mejora y cambio. Un ejemplo de este involucramiento, es el establecimiento de continuidades entre la escuela y otros agentes educativos, como la familia, para facilitar la coherencia entre contextos de desarrollo diferenciados, que a veces pueden ser contrapuestos (valores, prácticas, códigos) y que incluso pueden convertirse en barreras para el éxito educativo (Poveda, 2001). Una experiencia educativa coherente con lo anterior, es el proyecto "Shere-Room" llevado a cabo en diferentes barrios de la corona metropolitana de Barcelona con alumnado de grupos culturales minoritarios, en donde predomina la población gitana (Crespo et al., 2014). En "Shere-Room", se propone el diseño de una actividad que se realiza fuera de la escuela, buscando recuperar los intereses, motivaciones y formas de participación por parte del alumnado. Para ello, las técnicas utilizadas a la hora de generar ganas de aprender se basan en 
la negociación y colaboración constante con los participantes, sus familias y los líderes de la comunidad gitana, en el caso de la población romaní. De este modo, el conocimiento es sensible a la cultura familiar y comunitaria del alumnado. Se busca la incorporación de referentes y modelos nuevos, como pueden ser miembros de la comunidad, o estudiantes universitarios que ejerzan de mentores. Se trabajan prioritariamente competencias básicas tales como la comunicación, habilidades instrumentales, así como las competencias digitales a través de metodologías de aprendizaje colaborativo y la resolución de tareas. Este tipo de experiencia, es un precedente claro para el consecuente desarrollo de programas basados en los fondos de conocimiento, como los que se presentan a continuación.

\subsection{Fondos de conocimiento en una familia marroquí}

Un ejemplo de cómo aplicar los fondos de conocimiento familiares en la escuela ha sido la propuesta de Saubich y Esteban-Guitart (2011), en dónde se parte de la premisa que todas las familias más allá de su condición social o diversidad cultural y económica disponen de una suerte de destrezas, relaciones, habilidades y saberes que tienen su origen es sus prácticas socioculturales tales como el trabajo, aficiones, comunitarias o bien religiosas. En este caso, se pone el foco en una familia de origen marroquí, en sus prácticas y recursos familiares, para fomentar espacios de continuidad educativa en grupos culturales y socioeconómicos que habitualmente no están presentes en las instituciones escolares en relación a prácticas de enseñanza y aprendizaje (Poveda, 2001). En las visitas llevadas a cabo por los docentes a la familia, y a partir de una serie de técnicas etnográficas como entrevistas, identificación e inventario de artefactos familiares y su uso en el hogar, se detectaron una serie de fondos de conocimiento que fueron categorizados de la forma siguiente: a) localización geográfica, b) política, sociedad y creencias religiosas, c) animales de Marruecos, d) clima y vegetación marroquí, e) el mercado y la comida marroquí y f) el uso de la "henna" como decoración corporal. En esta experiencia, el profesor de ciencias sociales usó un dispositivo cultural relevante como el Qur'an para desplegar los objetivos de aprendizaje tales como diversidad de valores y prácticas en las sociedades, así como realidades geopolíticas actuales. La profesora de ciencias de la naturaleza usó el conocimiento sobre los animales y el clima en la zona del Sahara marroquí, por parte de la familia, para desarrollar una unidad didáctica sobre los fenómenos meteorológicos, la fauna y la flora. Además, hubo unidades que organizadas en proyectos (ámbitos temáticos) se desarrollaron de forma colectiva en grupos cooperativos. Este fue el caso de la unidad didáctica "comida marroquí" que contó con la búsqueda de información, conocimiento y compra de ingredientes culinarios y la posterior elaboración de los platos de forma conjunta. En ella se trabajaron: 1) competencias propias del ámbito matemático tales como resolución de problemas y razonamiento, 2) competencias relacionadas con conocimiento del medio tales como salud y equilibrio personal, 3) competencias vinculadas con el conocimiento artístico como imaginación y creatividad, 4) competencias referentes al ámbito de educación en valores, como dimensiones interpersonales y multiculturales. La modificación de las prácticas tradicionales de relación entre la familia y la escuela, y el hecho que los docentes se acerquen a los hogares de sus estudiantes como aprendices (González, Moll y Amanti, 2005), ha sido fundamental para avanzar hacia formas más diversas y equitativas de incluir a la familia dentro de las prácticas educativas escolares, sobretodo, para aquellas que asumen mayores cotas de desigualdad, injusticia social e invisibilidad. De este modo, el alumnado infrarrepresentado, como el de comunidades inmigrantes tales como la marroquí, experimentan menores discontinuidades cuando los docentes son capaces de reconocer su trasfondo familiar y su herencia cultural.

\subsection{El proyecto Girona: Fondos de conocimiento para empoderar a los hogares}

El proyecto Girona se desarrolló en diez centros próximos a la ciudad de Girona con alumnado y familias procedentes de África, Rumanía y Suramérica (Esteban-Guitart y Vila, 2013; Subero et al., 2015). A grandes rasgos, en las diferentes escuelas sondeadas se detectaron una serie de fondos de conocimiento que estuvieron relacionados con las siguientes temáticas, a saber: a) conocimientos y competencias multilingües, b) conocimientos de medicina y anatomía, c) religión, d) construcción, e) conocimientos deportivos, f) conocimientos musicales y artísticos, g) agricultura y horticultura, h) mantenimiento del hogar, i) economía. A partir de los ámbitos temáticos introducidos, se seleccionaron los siguientes fondos de conocimiento susceptibles de poder ser vehiculados hacia actividades educativas escolares. Este procedimiento se realizó dentro de los grupos de estudio. De este modo, del fondo de conocimiento "historia de Rumanía y sus formas de gobierno" se llevó a cabo la actividad de Trivial. Es decir, un concurso 
de preguntas sobre las diferentes formas de gobierno a lo largo de la historia y en la actualidad entre dos países como Rumanía y España. Esta actividad se desarrolló en la asignatura de historia en el curso de Bachillerato. A continuación, otro fondo de conocimiento que fue tenido en consideración fue "el cultivo de productos africanos", en especial las familias que procedían de Gambia y que tenían una gran experiencia en el cultivo y el conreo. La actividad propuesta fue el cultivo de una huerta con productos africanos que trabajó el tema del mundo vegetal. La actividad se desarrolló en el tercer curso de primaria y fue una actividad transversal.

De las experiencias del proyecto Girona, vamos a compartir un ejemplo ilustrativo vinculado a la construcción que se llevó a cabo en una escuela pública de la comarca del Pla de l'Estany (Girona) que contaba con una alta densidad de alumnado y familias inmigradas en dónde Marruecos, Gambia y Rumanía son las nacionalidades con mayor número. El proyecto se desarrolló en un aula de P5 (segundo ciclo de educación infantil) con una casuística de 22 alumnos y alumnas, de los cuales 9 de ellos eran extranjeros procedentes principalmente de países de áfrica subsahariana. Dada la gran cantidad de alumnado procedente de Gambia, se optó por escoger las familias de esta nacionalidad para llevar a cabo las visitas a los hogares. La relación inicial con las familias era cordial, pero esporádica. Los docentes, de este modo, realizaron una serie de visitas a los hogares de los alumnos objeto de estudio, en dónde llevaron a cabo una serie de entrevistas y anotaron, a través de registros observacionales, información sobre el barrio y elementos representativos del hogar. Además, las entrevistas recogieron información sobre las experiencias y trayectorias vitales familiares, la profesión y oficios de los padres, sus aficiones, las actividades que realizaban conjuntamente en familia, así como el interés por sus redes sociales y de confianza. A partir de estas informaciones, destacaron los siguientes ámbitos temáticos: 1) la importancia de la historia familiar de tránsito desde Gambia hacia España, 2) la importancia de tener familiares y amigos en el lugar de acogida y 3) la relevancia del trabajo del padre en la familia. El rol paterno fue significativo destacando como la construcción estaba presente en el contexto familiar a través de conversaciones, explicaciones cotidianas y experiencias de vida que los hijos e hijas tomaban como propias y relevantes.

Dentro de los grupos de estudio, se escogió la construcción como fondo de conocimiento óptimo para formar parte de las actividades escolares, ya que distintas familias tenían experiencias vinculadas con esta profesión. A continuación se llevó a cabo la unidad didáctica titulada "hago paredes, hago cimientos y sobre los andamios subo contento" en la que se trabajaron una serie de competencias tales como: a) la lengua (expresión oral, comprensión, uso de elementos narrativos y adivinanzas así como el trabajo de vocabulario referente al ámbito temático), b) el descubrimiento del entorno, c) tecnologías del aprendizaje y la comunicación (a través de software como el JClic y el uso y modificación de imágenes), d) lenguaje musical, e) creación artística y f) psicomotricidad. La unidad se constituyó por una serie de actividades incorporando elementos característicos de la construcción como: a) materiales y tipología de casas, b) cuento sobre las "casas del mundo" de Clémentine Sourdais, c) construcción de un diccionario bilingüe con vocabulario específico de la construcción y d) elaboración de un mural final abierto a las familias. A destacar, fue la participación de alguno de los padres entrevistados para ayudar en el desarrollo de las actividades y dejarse preguntar por el alumnado sobre su experiencia laboral. La incorporación de los fondos de conocimiento facilitó el cambio de expectativas iniciales de los docentes sobre las familias, facilitando el conocimiento de las culturas del alumnado y rompiendo estereotipos. Sin embargo, lo relevante de la propuesta es que fue más allá de ser un programa bienintencionado que proclamaba la necesidad de desarrollar procesos de igualdad y equidad en el ámbito discursivo, sino que a su vez llevó a cabo prácticas que buscaban equilibrar y reconocer el capital cultural de las familias infrarrepresentadas, en este caso las familias gambianas, materializando prácticas y dinámicas educativas explícitas en el aula.

\subsection{Familias gitanas y fondos de conocimiento, nuevas formas de participación}

Lorenzo-Moledo y colaboradores (2020) propusieron la incorporación de los fondos de conocimiento para fomentar la participación de las familias gitanas en las prácticas escolares de sus hijos. Esta tercera experiencia se llevó a cabo con familias y alumnado de origen romaní de educación primaria en Pontevedra. La experiencia se desarrolló en tres fases: 1) establecimiento de continuidades entre la familia y los docentes a través de agentes educativos de la comunidad, 2) elaboración de la unidad didáctica e 3) implementación de la unidad didáctica. 
Por lo que respecta a la metodología clásica de los fondos de conocimiento en que los docentes se acercan a los hogares de las familias para recoger los cuerpos y saberes acumulados, los autores incorporaron un tercer actor educativo en la variable con el objetivo de tener éxito a la hora de aproximarse a la población objeto de estudio y consolidar una participación sostenida de las familias a lo largo de todo el proceso. Este tercer agente educativo fue la Fundación Secretariado Gitano. De este modo, en comunidades culturales en que es más difícil el acercamiento a su contexto cultural cotidiano, la colaboración con el Secretariado Gitano permitió facilitar las relaciones entre la familia y la escuela y posibilitar un espacio de bienestar en que las familias gitanas pudieran sentirse acogidas, cómodas y fuera sencillo generar un clima de confianza óptimo para motivar su participación en la dinámica del proyecto. Durante tres meses se sucedieron los espacios con las familias a razón de adaptarse a su predisposición, necesidades y horario familiar. Así, durante sesiones de dos horas semanales, las autoras llevaron a cabo las dos primeras fases del proyecto. Como inciso, previo al inicio de la primera fase, se trabajó con el profesorado el enfoque teórico y metodológico de los fondos de conocimiento a través de su formación y presentación en diferentes sesiones.

La primera fase consistió en establecer los primeros espacios de corresponsabilidad entre las familias y los docentes en las que se reivindicó el papel de las familias para la escolarización y el éxito académico de sus hijos e hijas. Lo relevante es que, en vez de fomentar acciones educativas compensatorias en relación a las familias gitanas, se reconsideró la forma de relación con ellas para mostrar interés por los recursos socioculturales que poseen y transformarlos en conocimiento útil para ser incorporado en el contexto escolar. Además de conocer sus prácticas cotidianas, las autoras recogieron información importante sobre los tipos de discursos y usos comunicativos más relevantes, que ayudó al profesorado poder ajustar mejor la propuesta de intervención futura en la escuela. En el proceso de recogida de los datos, se hizo hincapié en las voces de las familias y el uso de sus historias de vida como recurso. En la segunda fase, las autoras prepararon las unidades didácticas y las prácticas educativas para llevarlas a cabo en la escuela con la participación explícita de las familias y el alumnado gitano. Es en este espacio dónde las autoras diseñaron, de forma conjunta, las actividades basadas en los fondos de conocimiento detectados.

Finalmente, en la tercera fase se incorporó el enfoque de los fondos de conocimiento a través de la unidad didáctica de relatos de vida familiar (Míguez, 2020). El proyecto se llevó a cabo con seis grupos de ciclo medio y ciclo superior de primaria. Las actividades contaron con seis actividades, a saber: 1) ¿Cómo es mi familia?, 2) ¿En qué se parecen y en qué se diferencian?, 3) ¿Cuál es mi historia familiar?, 4) Mi familia antes y ahora, 5) Relato autobiográfico. Esta última actividad, es la culminación de la unidad didáctica y consistió en la elaboración de una narración autobiográfica del alumnado junto a un miembro de su comunidad (madres y/o abuelas). Por narración se entiende una forma de construcción de la realidad, que tiene un significado relevante, y se genera a través de la expresión y la reconstrucción de vivencias, en relación con los otros, a través de la reflexión y mediante la comunicación o un discurso determinado (Bolívar, 2002). En la unidad didáctica, la narración consistió en la elaboración de algún suceso o acontecimiento familiar que se consideró relevante alrededor de cinco ejes: escuela, juventud, cultura, tiempo libre y oficios. Los docentes acompañaron el desarrollo de la actividad a partir de sugerencias y apoyos a lo largo del proceso de elaboración. Una vez elaborados los relatos, se leyeron al global del alumnado y se invitó a las madres y/o abuelas al aula como testimonios directos. El alumnado, a su vez, pudo formular preguntas o reflexiones sobre aquello que el relato les sugería o interpelaba.

Por lo anterior, se destaca la importancia de diseñar propuestas educativas inclusivas que reconozcan la realidad cultural gitana en las aulas y promuevan procesos de transformación social y éxito educativo en el alumnado a través de narrativas autobiográficas. Para ello, son necesarias nuevas formas de organizar el espacio escolar así como el uso de instrumentos educativos pertinentes, tales como el currículum o la participación de otros agentes educativos relevantes como la familia y la comunidad, aquí representada por la Fundación Secretariado Gitano. Entendemos que este tipo de proyectos basados en los fondos de conocimiento en que se legitima los recursos culturales y educativos de familias infrarrepresentadas, como la gitana, va en la línea de visibilizar y reconsiderar a dichos alumnos y alumnas como sujetos de derecho, avanzando hacia prácticas educativas socialmente justas. 


\section{Discusión y conclusiones}

Los tres proyectos presentados anteriormente, se valen del concepto de los fondos de conocimiento para originar nuevas formas de posicionarse en el trabajo educativo entre familias, docentes y estudiantes.

La primera experiencia, sobre el desarrollo de fondos de conocimiento referente a una familia marroquí, expone como a partir de diferentes técnicas, tales como el dibujo identitario, el círculo significativo o las experiencias de aprendizaje contextualizadas en las prácticas familiares registradas a través de las fotografías, se logra hacer emerger una serie de conocimientos susceptibles de ser incorporados en las prácticas escolares. Es a partir del reconocimiento de conocimientos tales como la lengua materna (el árabe), la cocina, la construcción o la religión que es posible avanzar hacia el diseño de unidades didácticas en la escuela en base a los fondos recogidos. Los resultados, a partir de entrevistas y grupos de discusión, indican que se avanza en la mejora de las relaciones familia y escuela. Es decir, se pasa de entender inicialmente la relación con las familias sobre prejuicios o estereotipos (o incluso desconocimiento), a establecer conocimientos personales compartidos. Además, el bilingüismo como fondo de conocimiento, es un importante recurso que permite la implicación y el desempeño académico, motivando al alumnado en el aprender y reafirmando sus identidades (Cummins, 2009). Existen experiencias educativas en el contexto estadounidense, como las de Schecter y Cummins (2003) que avanzan en la elaboración de "textos identitarios" bilingües, a partir de diferentes recursos lingüísticos y audiovisuales, para promover los conocimientos e identidades de los alumnos recién llegados o de origen inmigrante.

En relación a la segunda experiencia, referente a la elaboración de una unidad didáctica relacionada con la construcción, a partir de las visitas realizadas por los docentes a las familias gambianas, se detallan los siguientes resultados. En primer lugar, se destaca la mejora de las relaciones entre la familia y la escuela (además de con el territorio), ya que se rompen con los prejuicios preestablecidos y se facilita el conocimiento cultural por parte de los docentes. Estas continuidades favorecen la práctica profesional de los profesores en relación al alumnado, así como con la familia de los mismos. Las evidencias fueron recogidas en entrevistas en profundidad y cuestionarios a partir de un diseño pre-post test. La experiencia de las familias gambianas, tiene su antecedente en una experiencia educativa llevada a cabo en USA por Sandoval-Taylor (2005) en dónde la maestra, a partir de la recogida de los fondos de conocimiento en los hogares de alumnos de origen mexicano, descubrió que compartían la construcción como conocimientos familiares comunes. A partir de ello, la autora elaboró una serie de actividades educativas en que, partiendo de la construcción, pudo trabajar diferentes materias de una forma transversal. Similar a las reflexiones presentadas por Subero y colaboradores (2015), Sandoval-Taylor (2005) argumenta que, una vez participado en el programa, el profesorado modificó sus creencias previas alrededor de las familias inmigrantes o de minorías étnicas. Además, se hizo énfasis en la mejora de las relaciones entre docentes y familias basadas en la confianza mutua y el intercambio recíproco. Por lo que respecta a la práctica y currículum escolar, se evidenció la creación de innovaciones docentes a través de unidades curriculares que partieron de los fondos de conocimiento del alumnado y, finalmente, se hizo explícita una mejora en el rendimiento académico.

En tercer lugar, la experiencia de los fondos de conocimiento con familias gitanas avanza en el diseño y desarrollo de un programa de mejora de las relaciones familia y escuela, así como del rendimiento escolar a partir de la unidad didáctica basada en la elaboración de los relatos de vida familiar. Mediante el uso de técnicas cualitativas tales como herramientas etnográficas, entrevistas y diarios de campo se recoge una alta implicación y satisfacción por parte de las familias a lo largo de las sesiones del programa. En relación al alumnado, se destaca la creación de espacios de aprendizaje basados en el diálogo y el juego a partir de los fondos de conocimiento detectados. Por lo que respecta a los docentes, han creado consciencia de la necesidad de formarse hacia la mejora de la diversidad en el aula para el desarrollo de proyectos interculturales de calidad (Míguez, 2020). Por lo que hace referencia a los productos finales, tales como los relatos de vida, elaborados a dos manos entre las familias y el alumnado, con el apoyo y el acompañamiento de los docentes, se ha fomentado el desempeño académico del alumnado a través de una mayor motivación como creadores de conocimiento. Los relatos de vida se constituyen, de este modo, en fuente primara de recogida de los fondos de conocimiento, dejando en un segundo plano la visita de los docentes a los hogares de la familia (metodología tradicional del enfoque). Como ventajas, se hace hincapié en el interés por parte del alumnado a la hora de compartir sus vivencias familiares, el fortalecimiento de la identidad a través de la historia personal que supone el relato, el sentimiento de pertenencia y reconocimiento por 
parte de los demás en relación al grupo familiar (en este caso, el gitano), el uso de experiencias cotidianas en las que el alumnado es el protagonista como recurso educativo en el contexto escolar y la emergencia de voces y discursos alternativos, a través de la narrativa, que posibilitan nuevas formas de justicia social educativa.

Pese a sus pequeñas diferencias en relación al grupo de participantes, así como en la forma en que los docentes se acercan a las familias infrarrepresentadas y recogen los fondos de conocimiento, se mantiene la misma finalidad a lo largo de las diferentes propuestas educativas: en primer lugar, las prácticas educativas basadas en fondos de conocimiento combaten las actitudes racistas por parte de la cultura escolar hacia las familias inmigrantes, así como por parte de ellas en relación a los docentes y la escuela. En segundo lugar, son un acicate para la mejora de la formación docente alrededor de una estrategia educativa que se ha mostrado eficaz para gestionar la diversidad social y cultural del alumnado y sus familias. Finalmente, en tercer lugar, refuerzan los enlaces entre la escuela y la familia favoreciendo que ésta última pueda ser pedagógicamente reconocida en las prácticas escolares.

Además, se documentan de forma empírica los recursos de las familias y se hacen presentes en forma de recursos pedagógicos para orientarlos académicamente en la escuela convirtiéndose así, finalmente, en recursos para el aprendizaje. El valor añadido de los fondos es que no solamente mejoran las relaciones entre la familia y la escuela, sino que son capaces de modificar las percepciones y expectativas iniciales de uno y otro a partir de la investigación y la práctica sociocultural. La propuesta de los fondos de conocimiento permite considerar la educación en su contexto, a partir de variables sociales, históricas, económicas y culturales particulares; capitales válidos para ser comprendidos en la escuela y necesarios para fomentar mayores cotas de significación y sentido (Moll, 2014). Por una parte, las familias sienten que son partícipes del aprendizaje de sus hijos y no se ven cuestionadas por sus formas de aprender y desarrollarse en sus hogares. Por otra parte, los docentes tienen la oportunidad de saber de primera mano, a partir de herramientas y metodologías basadas en los fondos de conocimiento, qué y cómo aprenden las familias de su alumnado infrarrepresentados, comprendiendo mejor los hogares, las prácticas familias y los recursos culturales que en ellos albergan. De este modo, los docentes son capaces de convertirse en una figura capaz de acompañar a su alumnado en el sentido más amplio de la palabra. Acompañar significa "estar o ir en compañía del otro, existir junto al otro, participar en sus sentimientos" (Tarabini, 2020, p.152). A partir de dicha inmersión cultural, es mucho más fácil generar innovaciones educativas que promuevan nuevos retos educativos, siendo sensibles a las historias sociales y los saberes cotidianos del alumnado.

Sin embargo, como toda práctica educativa, las tres experiencias expuestas, así como el enfoque de los fondos de conocimiento en su conjunto, conllevan ciertas dificultades o limitaciones que se exponen a continuación. En un primer momento, a lo largo de los tres proyectos, se hace extensible la necesidad de mayor tiempo, esfuerzo y trabajo no solamente para conocer con mayor profundidad y rigor al alumnado y sus familias, sino para elaborar y desarrollar las unidades didácticas en el contexto escolar con la calidad pedagógica que se requiere. Además, en gran parte de las experiencias, también es necesario cursos de aproximación y familiarización con el enfoque de los fondos de conocimientos por parte de los docentes para poder dotarles de un marco teórico, metodológico y estrategias pertinentes para llevarlas a cabo en sus experiencias educativas. En segundo lugar, la diversidad en las aulas, en el contexto educativo español, suele incorporar distintos entornos sociales y culturales, hecho que dificulta poder centrarse en un grupo étnico o cultural específico y generalizar en el aula los fondos de conocimiento detectados a partir de unidades didácticas. En tercer lugar, es importante no caer en una idea de cultura, o de práctica cultural que se base en aspectos folclóricos, generables o permanentes (Gonzalez et al., 2005). De este modo, se corre el riesgo de no profundizar sobre las experiencias familiares en particular, evitando así recoger fondos de conocimientos reales y significativos. A pesar de estas limitaciones o dificultades, es necesario tener presente los avances que supone el enfoque de los fondos de conocimiento como estrategia novedosa, socialmente justa y contextualizada en la gestión de la diversidad e interculturalidad en las aulas.

A modo de conclusión, podemos considerar los fondos de conocimiento como alternativas pedagógicas a las prácticas educativas que mantienen la indiferencia (Lingard y Keddie, 2013) sobre el alumnado infrarrepresentado y que acaban por normalizar la injustica y la desigualdad como prácticas cotidianas comunes o males menores. La propuesta de los fondos de conocimiento representa, más que nunca, una forma de entender las prácticas pedagógicas coherente con propuestas disruptivas, democráticas, decoloniales, 
antirracistas, orientadas hacia la justicia social y comprometidas con un proceso continuo de reflexión y mejora permanente (Iglesias et al., 2020; Belavi y Murillo, 2020).

\section{Referencias}

Bauman, Z. (2017). La cultura en la modernidad líquida. FCE.

Belavi, G. y Murillo, F. J. (2020). Democracia y justicia social en las escuelas: Dimensiones para pensar y mejorar la práctica educativa. REICE. Revista Iberoamericana Sobre Calidad, Eficacia Y Cambio En Educación, 18(3), 5-28. https://doi.org/10.15366/reice2020.18.3.001

Bolívar, A. (2002). ¿De nobis ipsis silemus?: Epistemología de la investigación biográfico-narrativa en educación. Revista electrónica de investigación educativa, 4(1), 1-26.

Castells, M. (1998). La era de la información: Economía, sociedad y cultura. Alianza.

Crespo, I., Lalueza, J. L., Lamas, M., Padrós, M. y Sánchez-Busqués, S. (2014). El proyecto “Shere Room”. Fundamentos de una comunidad de prácticas para la inclusión educativa de grupos culturales minoritarios y en riesgo de exclusión social. Psicología, Conocimiento y Sociedad, 4(2), 138 -162.

Cummins, J. (2009). Fundamental psychological and sociological principles underlying educational success for linguistic minority students. En T. Skutnabb-Kangas, R. Phillipson, A. K. Mohanty y M. Panda (Eds.), Social justice through multilingual education. (pp. 19-35). Multilingual Matters.

Darling-Hammond, L. (2010). The flat world and education: How America's commitment to equity will determine our future. Teachers College Press.

Erchick, D. B. y Tyson, C. A. (2013). Social justice in a mathematics education professional development program: Coach growth progressions. American International Journal of Social Science, 2(8), 1-9.

Esteban-Guitart, M. y Ratner, C. (2011). A macro cultural psychological theory of identity. Journal of Social Distress and Homelessness, 2O(1), 1-22. https://doi.org/10.1179/105307811805365016

Esteban-Guitart, M. y Vila, I. (2013). Experiencias en educación inclusiva: Vinculación familia, escuela y comunidad. Horsori.

Esteban-Guitart, M., Serra, J.M. y Llopart, M. (2018) The role of the study group in the funds of knowledge approach, Mind, Culture, and Activity, 25(1), 216-228. https://doi.org/10.1080/10749039.2018.1448871

Esteban-Guitart, M., Lalueza, J. L., Zhang-Yu, C. y Llopart, M. (2019). Sustaining students' cultures and identities. A qualitative study based on the funds of knowledge and identity approaches. Sustainability, 11(12), 1-12. https://doi.org/10.3390/su11123400

Freire, P. (1998). Pedagogy of the oppressed. Continuum.

Gay, G. (2018). Culturally responsive teaching. Theory, research, and practice. Teachers College Press.

Gonzalez, N., Moll, L. y Amanti, C. (Coord.). (2005). Funds of knowledge: Theorizing practices in households, communities, and classrooms. Routledge.

Han, B. (2014). En el enjambre. Herder.

Hollie, S. (2018). Culturally and linguistically responsive teaching and learning. Shell Educational.

Iglesias-Vidal, E., González-Patiño, J., Lalueza, J. L. y Esteban-Guitart, M. (2020). Manifiesto en tiempos de pandemia: Por una educación crítica, intergeneracional, sostenible y comunitaria. Revista Internacional De Educación Para La Justicia Social, 9(3), 181-198. https://doi.org/10.15366/riejs2020.9.3.010

Ladson-Billings, G. (1995). Toward a theory of culturally relevant pedagogy. American Educational Research Journal, 32(1), 465-491. https://doi.org/10.3102/00028312032003465

Lalueza, J. L. y Crespo, I. (2009). Voices in the “Gipsy Developmental Project”. Mind, Culture and Activity, 16(1), 263280. http://dx.doi.org/10.1080/10749030802601304 
Lingard, B. y Keddie, A. (2013). Redistribution, recognition and representation: Working against pedagogies of indifference. Pedagogy, Culture and Society, 21(3), 427-447. https://doi.org/10.1080/14681366.2013.809373

Llopart, M. y Esteban-Guitart, M. (2018). Funds of knowledge in 21st century societies: Inclusive educational practices for under-represented students. A literature review. Journal of Curriculum Studies, 50(2), 145-161. https://doi.org/10.1080/00220272.2016.1247913

Lorenzo-Moledo, M., Míguez-Salina, G. y Cernadas-Ríos, F. (2020). ¿Pueden contribuir los fondos de conocimiento a la participación de las familias gitanas en la escuela? bases para un proyecto educativo. Teoría de la Educación, 32(1), 191-211. https://doi.org/10.14201/teri.21299

MECD. (2018). Datos y cifras curso escolar 2018/2019. Madrid.

Miguez, G. (2020). Los fondos de conocimiento y la optimización del aprendizaje escolar en la infancia gitana [Tesis Doctoral, Universidad de Santiago de Compostela]. http://hdl.handle.net/10347/24013

Moll, L. C. (2014). L. S. Vygotsky and education. Routledge.

Moll, L. C. (2019). Elaborating funds of knowledge: Community-oriented practices in international contexts. Literacy Research: Theory, Method, and Practice, 68(1), 130-138. https://doi.org/10.1177/2381336919870805

Nussbaum, M. C. (2015). Philosophy and economics in the capabilities approach: An essential dialogue. Journal of Human Development and Capabilities, 16(1), 1-14. https://doi.org/10.1080/19452829.2014.983890

OECD. (2016). PISA 2015 Results: Excellence and equity in education. OECD.

OECD. (2018). The resilience of students with an immigrant background: Factors that shape well-being. OECD.

OECD. (2019). PISA 2018: Insights and interpretations. OECD.

Pardo, J. L. (2016). Estudios del malestar. Políticas de la autenticidad en las sociedades contemporáneas. Anagrama.

Poveda, D. (2001). La educación de las minorías étnicas desde el marco de las continuidades-discontinuidades familia-escuela. Gazeta de Atropología, 17, 1-17.

Ratner, C. (2011). Macro cultural psychology, the psychology of oppression, and cultural psychological enrichment. En P. Portes y S. Salas (Eds.), Vygotsky in 21st century society: Advances in cultural historical theory and praxis with non-dominant communities (pp.87-112). Peter Lang.

Rios-Aguilar, C., Kiyama, J. M., Gravitt, M. y Moll, L. C. (2011). Funds of knowledge for the poor and forms of capital for the rich? A capital approach to examining funds of knowledge. Theory and Research in Education, 9(2), 163-184. https://doi.org/10.1177/1477878511409776

Sandoval-Taylor, P. (2005). Home is where the heart is; a funds of knowledge-based curriculum module. En C. Amanti, N. Gonzalez y L. Moll (Eds.), Funds of knowledge: Theorizing practices in households, communities, and classrooms (pp. 153-165). Routledge.

Sassen, S. (2010). Territorio, autoridady derechos. Katz.

Saubich, X. y Esteban-Guitart, M. (2011). Bringing funds of family knowledge to school: The living Morocco project. Multidisciplinary Journal of Educational Research, 1(1), 79-103. https://doi.org/10.4452/remie.2011.04

Schecter, S. R. y Cummins, J. (2003). Multilingual education in practice: Using diversity as a resource. Heinemann.

Standing, G. (2013). El precariado. Una nueva clase social. Pasado y Presente.

Subero, D., Llopart, M., Vila, I. y Esteban-Guitart, M. (2015). La capitalización educativa de los fondos de conocimiento e identidad. El 'Proyecto Girona'. Papeles de Trabajo sobre Cultura, Educación y Desarrollo Humano, 11(2), 69-73.

Subero, D., Vila, I. y Esteban-Guitart, M. (2015). Some contemporary forms of the funds of knowledge approach. Developing culturally responsive pedagogy for social justice. International Journal of Educational Psychology, 4(1), 33-53. https://doi.org/10.4471/ijep.2015.02

Tarabini, A. (2020). ¿Para qué sirve la escuela? reflexiones sociológicas en tiempos de pandemia global. Revista de Sociología de la Educación, 13(2), 145-155. https://doi.org/10.7203/RASE.13.2.17135 
UNESCO. (2000). Marco de acción de Dakar: Educación para todos. Cumplir nuestros compromisos comunes (con los seis marcos de acción regionales). Foro mundial sobre la educación. UNESCO.

Valencia, R. (2010). Dismantling contemporary deficit thinking. Educational thought and practice. Taylor and Francis.

Vélez-Ibáñez, C. y Greenberg, J. (1992). Formation and transformation of funds of knowledge among U.S.-Mexican households. Anthropology and Education Quarterly, 23(1), 313-335.

https://doi.org/10.1525/aeq.1992.23.4.05x1582v

\section{Breve CV del autor}

\section{David Subero}

Doctor en Psicología (Programa de Doctorado Interuniversitario en Psicología de la Educación Coordinado por la Universidad de Barcelona). Profesor asociado por la Universidad de Girona. Miembro del grupo de investigación Cultura y Educación en la misma universidad, cuyas principales líneas son: Relaciones familia, escuela y comunidad; Mecanismos psicosociales y construcción de la identidad en contextos de diversidad social y cultural; Identificación y análisis de trayectorias y experiencias significativas de aprendizaje. Autor de diferentes capítulos de libro, artículos científicos y contribuciones a congresos. Email: david.subero@udg.edu

ORCID ID: https://orcid.org/0000-0002-4737-0047 